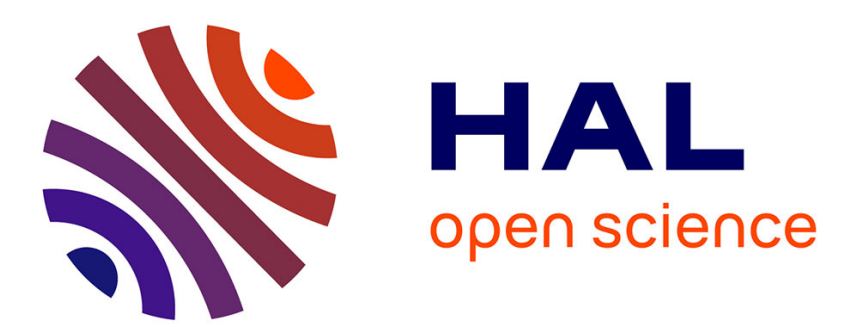

\title{
Adaptive compensation of High Frequency Memory Effects of a Realistic Amplifier in an OFDM System
}

Ammar Chaker, Myriam Ariaudo, Inbar Fijalkow, Sylvain Traverso, Jean-Luc

Gautier

\section{- To cite this version:}

Ammar Chaker, Myriam Ariaudo, Inbar Fijalkow, Sylvain Traverso, Jean-Luc Gautier. Adaptive compensation of High Frequency Memory Effects of a Realistic Amplifier in an OFDM System. 2007. hal-00137754

\section{HAL Id: hal-00137754 \\ https://hal.science/hal-00137754}

Preprint submitted on 21 Mar 2007

HAL is a multi-disciplinary open access archive for the deposit and dissemination of scientific research documents, whether they are published or not. The documents may come from teaching and research institutions in France or abroad, or from public or private research centers.
L'archive ouverte pluridisciplinaire HAL, est destinée au dépôt et à la diffusion de documents scientifiques de niveau recherche, publiés ou non, émanant des établissements d'enseignement et de recherche français ou étrangers, des laboratoires publics ou privés. 


\title{
Adaptive Compensation of High Frequency Memory Effects of a Realistic Amplifier in an OFDM System
}

\author{
Ammar Chaker, Myriam Ariaudo, \\ Inbar Fijalkow \\ Sylvain Traverso, Jean-Luc Gautier, \\ ECIME laboratory, ENSEA/UCP \\ chaker@ensea.fr \\ ETIS laboratory, \\ ENSEA, 6 avenue du Ponceau, 95014 Cergy-Pontoise - France.
}

\begin{abstract}
In addition to the power nonlinearity, the High Frequency (HF) memory effects of the Power Amplifier (PA) have a distorting influence even in the linear zone. The objective of this paper is to present a simple way to compensate for these memory effects in an Orthogonal Frequency Division Multiplex (OFDM) system. In order to represent the PA with HF memory effects in a realistic way, we use a circuit-level description by carrying out mixed digital signal processing and Radio Frequency (RF) circuit cosimulation. Our solution is based on the Wiener model of the PA and takes benefit of the cyclic prefix. In addition, the new compensation method takes into account the variation of the HF memory characteristics with the power. We illustrate the performance of our method and the accuracy of the Wiener model by means of digital/RF cosimulation.
\end{abstract}

Index Terms - HF Memory effects, Nonlinear Power Amplifier, Orthogonal Frequency Division Multiplex (OFDM).

\section{Introduction}

Recently, a worldwide convergence has occurred for the use of the Orthogonal Frequency Division Multiplex (OFDM) modulation for high data rates transmission. OFDM has been adopted in many standards like Digital Audio Broadcasting (DAB), Digital Video Broadcasting (DVB), High PErformance Radio Local Area Network type 2 (HIPERLAN/2) and MultiBands Ultra Wide Band (MB-UWB) [1]. Its most useful attractive feature is its high spectral efficiency (due to the orthogonality of the subcarriers) and it is particularly suited for frequency selective channels. Thanks to a cyclic prefix, this technique transforms a frequency-selective channel into a set of flat fading channels. The receiver complexity is reduced to a DFT processing and only one tap scalar equalization per carrier [1]. However, since $N$ subcarriers weighted by complex symbols coming from a particular mapping are summed, the signal has a large dynamic range. This presents two major drawbacks: a large dynamic for the analog/numeric conversion and a system sensitivity to the nonlinearities of the RF frontend components, in particular the power amplifier (PA) at the transmitter.

Indeed, the amplifier behavior varies according to the input power [2]. At high power, the amplifier has AM/AM nonlinearity that involves clipping of the signal (saturation) and mixing of the subcarriers (intermodulation), and AM/PM nonlinearity which introduces nonlinear phase shift. These effects are well known and there are well-established modeling techniques for narrowband system level. But, these techniques are not sufficiently accurate for wideband communication systems.

In fact, the PA, the output response at a given instant depends not only on the input signal at the same time instant but also the input signal at preceding instants over a limited duration, leading to so-called "High Frequency memory effect". Spectrally, this can be expressed as a spectral distortion of the signal which introduces performance degradation in wideband systems even in the linear zone of the PA (low input power) [3] [4].

In this paper, we study the impact of a PA on an OFDM signal in order to compensate for the HF memory effects as simply as possible. Wiener model which represents simply the PA helps us to derive an initial compensation method to compensate for $\mathrm{HF}$ memory effects. This is done at the transmitter before the OFDM modulator and is possible thanks to a long enough cyclic prefix [5]. Then, we improve it by taking into account the variation of the HF memory characteristics with the input power. To validate our method in a realistic case, we use a cosimulation of the electronic circuit of the PA, thereby, the HF memory effects of the PA are taken into account by performing circuit-level simulation of the amplifier, which yields highly accurate simulations close to what can be observed using real amplifier measurements. We denote this representation by realistic amplifier.

The remainder of the paper is organized as follows. In section II, we present a brief description of the OFDM system and the PA. Next, we show results concerning the influence of the HF memory of the realistic PA on a 
16-QAM based OFDM signal by doing cosimulation in the Advanced Design System (ADS) software. Cosimulation permits a behavioral simulation of the system along with a circuit simulation of the amplifier. Finally we show in section III how it is possible in an OFDM system to compensate for the HF memory effects with adaptation to the input power.

\section{System Description}

\subsection{OFDM System}

In an OFDM system the whole available bandwidth is divided into $N$ sub-bands. $N$ data symbols coming from a particular mapping (16-QAM in our simulations) are modulated on the $N$ corresponding subcarriers, which are orthogonal to each other thanks to the Inverse Discrete Fourier Transform (IDFT).

Before the pulse shaping, a cyclic prefix is inserted at the beginning of each OFDM symbol to "absorb" the echo delays of the multipath channel and to allow an easy equalization [1]. The signal is then carried to the Radio Frequency (RF) and amplified for the transmission. A block diagram of the simulated system is presented in Figure 1.

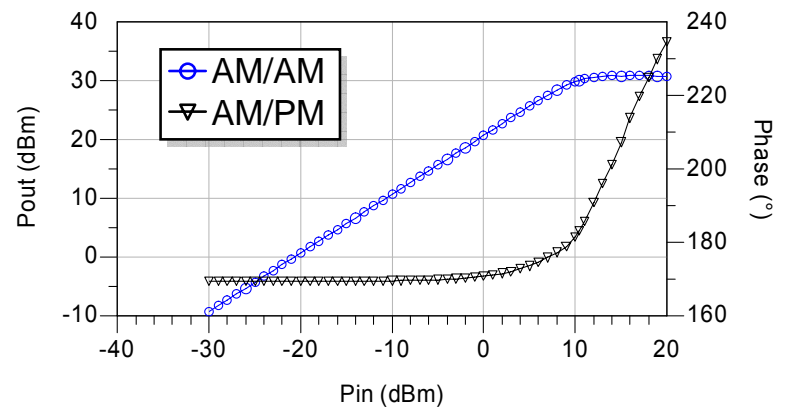

Figure 2: Amplitude and phase characteristics of the PA.

The HF memory of the PA causes gain variation and phase variation with respect to the frequency for a given input power. Figure 3-(a) \& (b) show gain and phase variation respectively for several average input powers covering the range -30 to $10 \mathrm{dBm}$. We notice that all gain and phase curves are identical in the range -30 to $10 \mathrm{dBm}$. The curves have a varying behavior above this range ( -10 to $10 \mathrm{dBm})$.

To observe meanly the HF memory effects on an OFDM system, the whole transmission chain is simulated in the ADS software and a cosimulation is performed. All the components except the PA are ideal

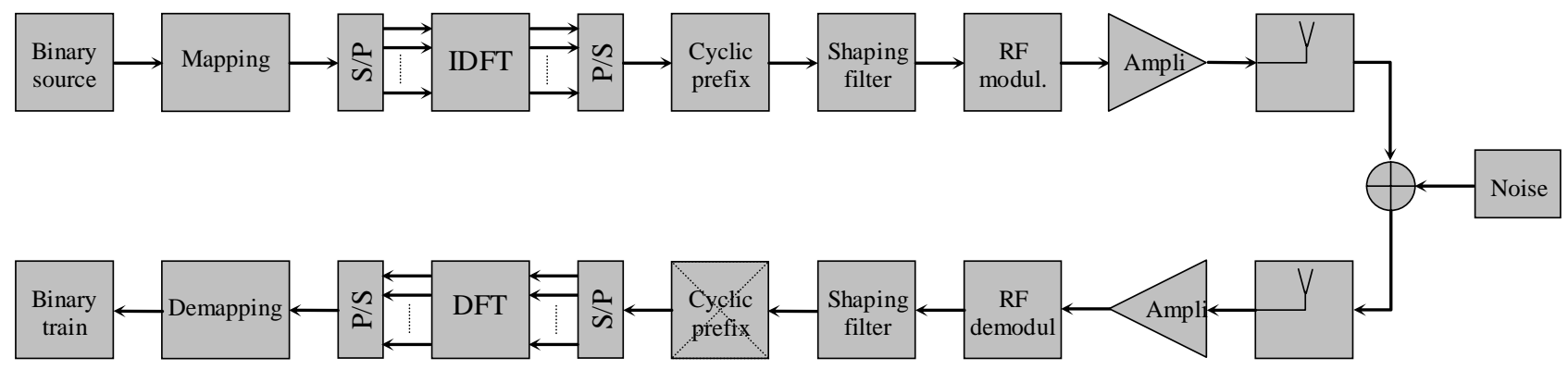

Figure 1: Block diagram of the transmission chain.

\subsection{Power Amplifier and HF Memory Effects}

The PA used in the system is designed in the RF circuit part of the simulation software. It is a two stages PA with a small signal gain of $22 \mathrm{~dB}$ at the central frequency of $5.2 \mathrm{GHz}$ and a $1 \mathrm{~dB}$ compression point of about $31 \mathrm{dBm}$. AM/AM and AM/PM characteristics are shown in Figure 2. and expressed by a behavioral model in the digital part of the software. The realistic amplifier's circuit is simulated in the RF part using the envelope transient method. It is excited by a 16-QAM based OFDM signal with 64 subcarriers of $20 \mathrm{MHz}$ bandwidth, and central carrier frequency $5.2 \mathrm{GHz}$ (Hiperlan2 is used as an example).

To quantify the degradation of the constellation at the receiver due to these effects, it is usual to evaluate the EVM (Error Vector Measurement) characteristic. It describes the difference between the ideal and real points of the constellation. Figure 6 shows the EVM result: we can see the effects of HF memory especially for weak input powers. For high power, the HF memory effects are hidden by the nonlinearity and saturation. 


\section{Compensation of the HF Memory Effects}

\subsection{PA Modeling}

The Wiener model is a common way to represent nonlinear systems with memory as a linear subsystem with memory followed by a memoryless nonlinear subsystem [6]. Some studies have shown that the Wiener model can be well suited to represent the PA, see [7]. In this model, memory effects, which can be expressed as the frequency dependency of the gain, are separated from the power nonlinearities.

In an OFDM system, the impulse response of the PA will not introduce Inter-Symbol Interference as long as the cyclic prefix is long enough. In practice, the impulse response of the PA is short and easily exceeded by the cyclic prefix.

(a)
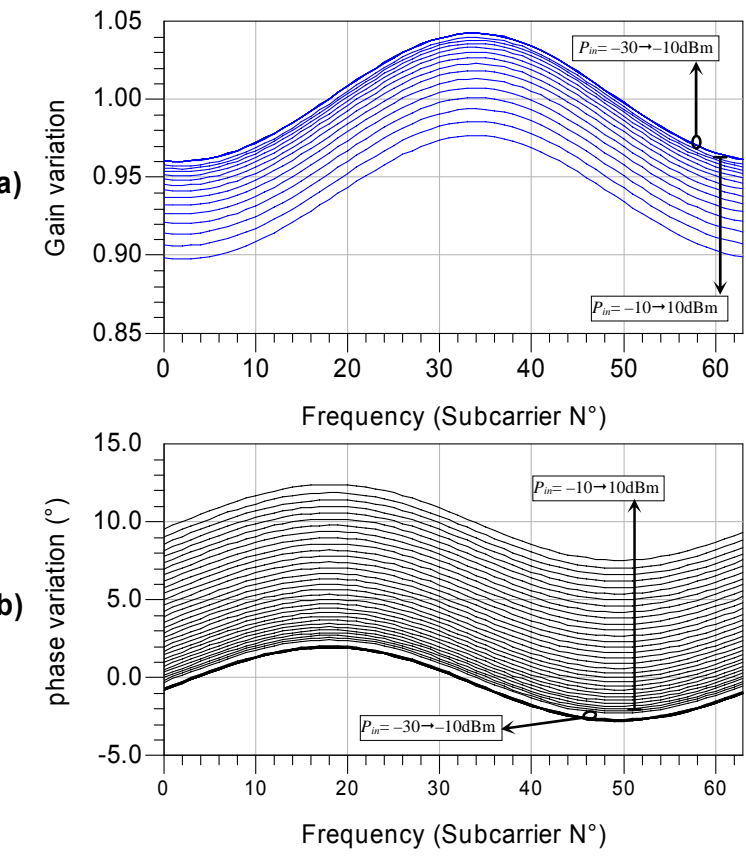

Figure 3: (a) gain and (b) phase variation due to memory effects.

\subsection{Memory Effects Compensation}

The insertion of the cyclic prefix, as in most OFDM systems, changes a linear convolution into a cyclic convolution as long as the length of cyclic prefix is longer than the impulse response [1]. A well-known property of the DFT is that the cyclic convolution in time domain results in a multiplication in the frequency domain. This is usually used to simplify equalization in
OFDM systems.

In our previous publication [5], we have proved that the use of the Wiener model for the amplifier leads to a simple and efficient compensation method in the case of the OFDM transmission. HF memory effects could be seen as a filter with a frequency response $H($ freq) So it is possible to "equalize" this effect simply by multiplying the IDFT inputs by $1 / H($ freq $)$.

The complex values of this $H($ freq) can be obtained directly from one of the gain and phase variation curves shown in Figure 3. An example at $0 \mathrm{dBm}$ of input power is presented in Figure 4.

(a)
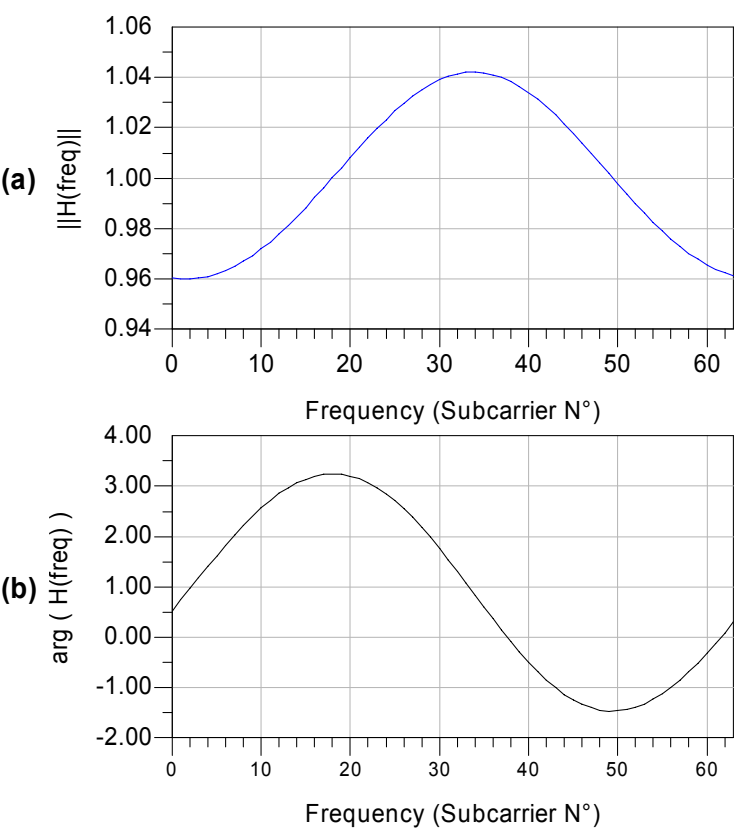

Figure 4: (a) gain and (b) phase variation due to memory effects for input power $=0 \mathrm{dBm}$.

This method can be applied only if the cyclic prefix is long enough, and its efficiency depends on the linearity of the components between the IDFT and the PA and the accuracy of Wiener model. Figure 5 shows the proposed architecture.

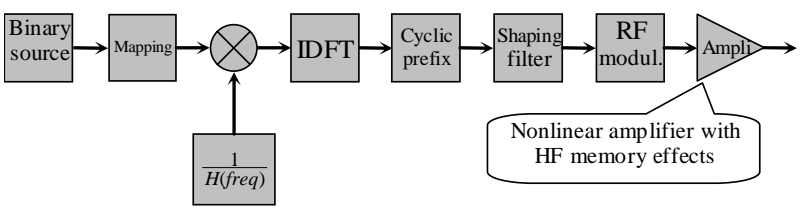

Figure 5: Proposed architecture to compensate for HF memory effects in OFDM system.

The result of this HF memory effects compensation method of is perfect in -30 to $-10 \mathrm{dBm}$ range where the EVM is reduced to zero as shown in Figure 6. This good 
compensation shows the interest of our method and the accuracy of the Wiener model at the same time.

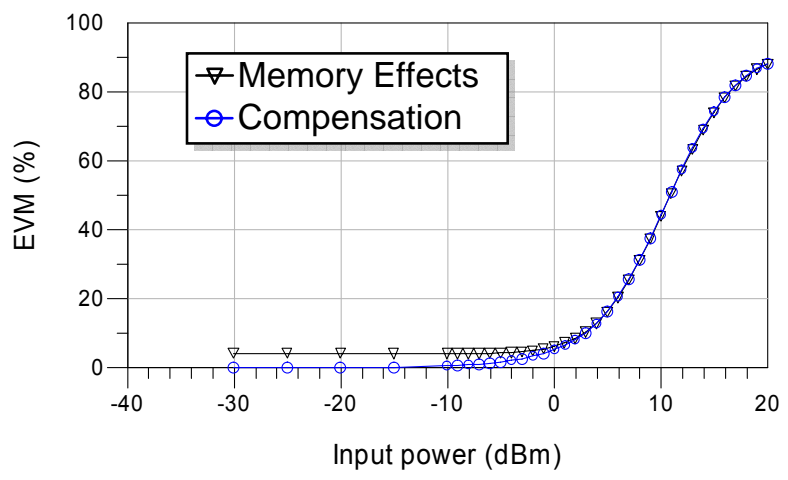

Figure 6: influence of memory effects and its compensation on the EVM.

As the memory effects behavior is constant in the -30 to $-10 \mathrm{dBm}$ range, compensation method is efficient. Above this range the behavior varies with input power. That is why the deterioration of the EVM performance caused by HF memory effects is almost entirely compensated for low input powers. However, the method yields no improvement for high input powers.

To improve the compensation performance for the high power zone, compensation function must vary as the power changes. in adaptive manner which is what we propose in the next section.

\subsection{Adaptive Compensation}

Indeed, as we can see in Figure 3, memory effects (gain and phase variation) is different for each power level that imposes the coefficients of $H$ (freq) to vary with the input power. The coefficients of the compensation function should be $H\left(P_{i n}, f r e q\right)$, with $P_{\text {in }}$ the mean input power of the amplifier for each frequency.

Thus, it is very important to be able to compensate with the appropriate coefficients, depending simultaneously on the frequency and the power. The new transmitter that we propose in this paper includes this property.

In order to perform compensation; first, the memory effects curves (gain and phase variation curves) are normalized in order to not to modify the average power of the OFDM signal. This is simply by dividing each gain curve by its mean value giving $H_{\text {norm }}\left(P_{\text {in }}\right.$,freq $)$ curves presented in Figure 7-(a). All of the mean values give the classical curve of gain shown in Figure 7-(b). Then the complex values of the function $1 / H_{\text {norm }}\left(P_{\text {in }}\right.$,freq $)$ are calculated and stored in an indexed table. (a)

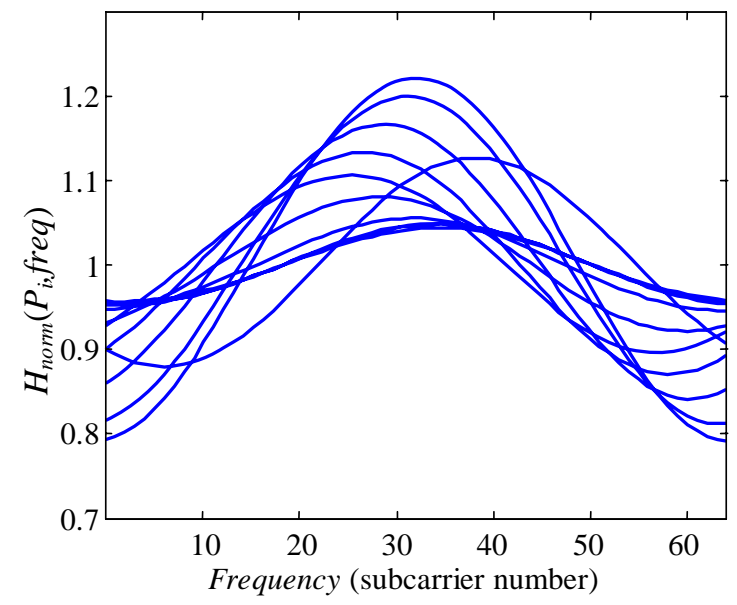

(b)

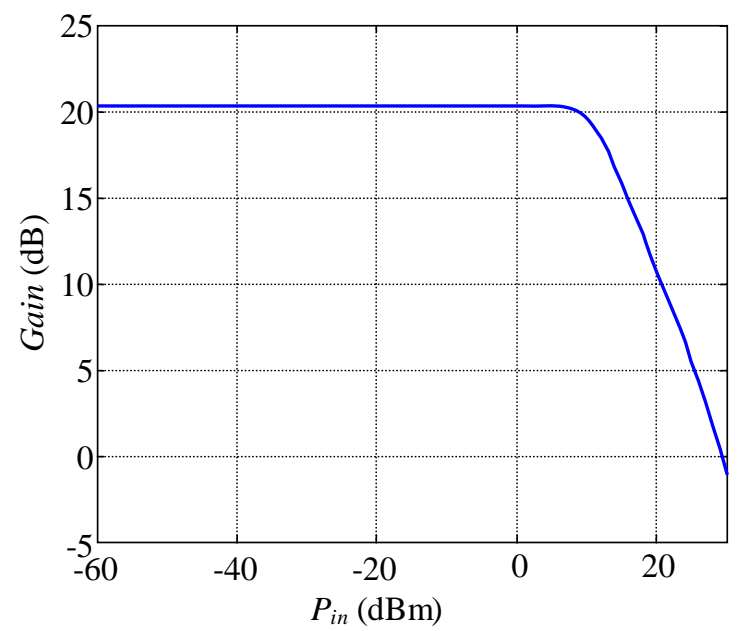

Figure 7: (a) normalized gain variation curves giving (b) the classical gain curve of the PA.

To choose the appropriate coefficients it is necessary to know the power transmitted by each subcarrier. In the case of an OFDM signal, each subcarrier is affected by a symbol coming from the initial mapping (16-QAM in our example) for which the power is well known. As our compensation method is applied before the IDFT, it affects directly the IDFT inputs (16-QAM symbols) by multiplying them by the appropriate coefficient depending on the 16-QAM symbol power.

It is also easy to determine the 16-QAM symbol power (only three possible values) and to extract the coefficient $H_{\text {norm }}\left(P_{\text {in }}\right.$,freq $)$ from an indexed table corresponding to the appropriate subcarrier and the appropriate power. Figure 8 shows an example of how to determine the $H_{\text {norm }}\left(P_{i}, f_{k}\right)$ function. Supposing that the power of the first QAM symbol on the first subcarrier is $\left(P_{1}\right)$, then the appropriate coefficient will be $H_{\text {norm }}\left(P_{1}, f_{1}\right)$, the second QAM symbol power on the second subcarrier is $\left(P_{3}\right)$, the coefficient is $H_{\text {norm }}\left(P_{3}, f_{2}\right)$ and so on. The inverse operation $\left(1 / H_{\text {norm }}\left(P_{i}, f_{k}\right)\right)$ is applied to obtain the function that will multiply the QAM sequence before the IDFT. 




Figure 8: $H_{\text {norm }}\left(P_{\text {in }}\right.$, freq $)$ calculation for adaptive compensation.

Taking into account the variation of the coefficients with the power leads to an improvement in the compensation performance seen on the EVM curve in Figure 9. We observe an important gain especially in the nonlinear zone which indicates that part of the nonlinearities is compensated for. Moreover, even if we have not shown the phase variation, we claim that this method allows correcting the variation of the phase with frequency and power. The AM/PM conversion is also partially corrected.

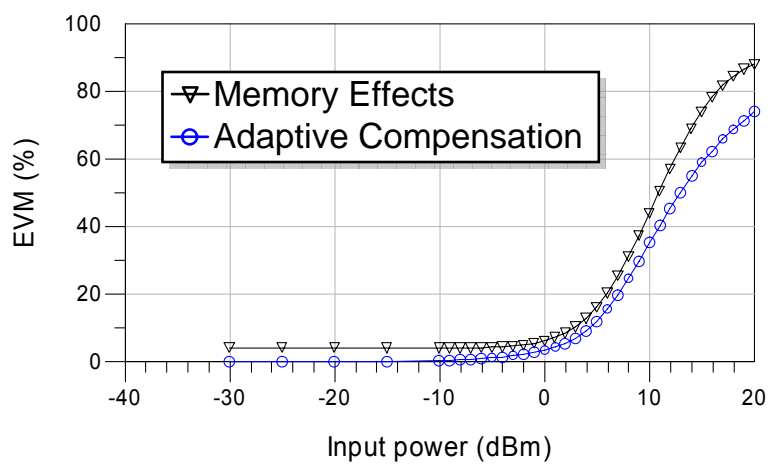

\section{Figure 9: Influence and compensation of HF} memory effects in terms of EVM.

Concerning the physical implementation of this method, we think that it is not a problem to evaluate a symbol power (quantization), memorize a coefficients table, choose the appropriate coefficient and do a multiplication. These operations can be implemented in an existing DSP without increasing much the complexity and the consumption.

\section{Conclusion}

The HF memory of the PA affects the transmission performance especially for broadband systems. Its effects on an OFDM system are studied using cosimulation of a realistic PA and a system level transmission chain. We have proposed and validated a method to compensate for the HF memory effects of Pas in OFDM systems. Moreover, as the PA frequency response $H($ freq $)$ varies with the input power of the PA, an improvement has been proposed by adapting the compensation coefficients with respect to the input power. The proposed new transmitter perfectly compensates for the flaws resulting from memory effects and the correction is easily performed at the transmitter thanks to a long enough cyclic prefix.

\section{References}

[1] R. Van Nee, R. Prasad, "OFDM for multimedia wireless communications," Artech House Publishers, 2000.

[2] S. C. Cripps, "RF Power Amplifiers for Wireless Communications," Artech House Publishers, 1999.

[3] T. T. Ha, "Solid-State Microwave Amplifier Design,” A Wiley-Interscience Publication,1981.

[4] H. Ku, M. D. McKinley, J. S. Kenney, “Quantifying Memory Effects in RF Power Amplifiers," IEEE transactions on Microwave Th. and Tech., VOL. 50, NO. 12, Dec. 2002.

[5] A. Chaker, M. Ariaudo, I. Fijalkow, J.-L Gautier, "Pre-compensation of the frequency-dependance of a non-linear amplifier in a multi-carrier transmission," IEEE International Conference on Communications ICC'04, June 2004, Paris.

[6] W. Greblicki, "Nonparametric Identification of Wiener Systems," IEEE Tran. On Information Theory, Vol. 38, No. 5, pp. 1487-1493, Sep. 1992.

[7] F. Launay, Y. Wang, S. Toutain, T. Reyverand, D. Barataud, J.-M. Nebus, R. Quere, "New method on wideband power amplifier modeling taking into account frequency-dependent behavior," EuMC 2001, London. 